\title{
Effect of sustained virologic response on the incidence of hepatocellular carcinoma in patients with HCV cirrhosis
}

\begin{abstract}
Background and objectives: Evidence suggests that sustained virologic response to interferon treatment decreases incidence of hepatocellular carcinoma in patients with hepatitis $C$ virus cirrhosis. This study was designed to compare the incidence of hepatocellular carcinoma among cirrhotic patients exposed to interferon based treatment with or without achieving a sustained virological response, in order to evaluate the role of interferon itself in the prevention hepatocellular carcinoma. Methods: A cohort of 85 patients with compensated hepatitis C cirrhosis was followed after treatment with interferon and ribavirin. Sustained virological response was defined as negative polymerase chain reaction assay 24 weeks after the end of treatment. Patients were followed every 6 months with ultrasound and alpha-fetoprotein. Hepatocellular carcinoma was diagnosed by the finding of a focal liver lesion greater than $2 \mathrm{~cm}$ with arterial hypervascularization on two imaging techniques and/or by liver biopsy. Results: The mean follow-up time was $32.1 \pm 20$ months for patients who achieved a sustained virological response and $28.2 \pm 18$ months among 47 patients $(55 \%)$ without SVR. Hepatocellular carcinoma was diagnosed in $1(3 \%)$ vs. 8 (17\%) responders and non responders respectively $(p=0.02)$. Conclusion: Patients with cirrhosis due to hepatitis $C$ virus who achieved sustained virological response had significantly lower incidence of hepatocellular carcinoma when compared to those without treatment response. Interferon treatment without achieving sustained virological response does not seem to protect against hepatocellular carcinoma.
\end{abstract}

Keywords: hepatocellular carcinoma, HCV interferon, sustained virologic response, cirrhosis.

[Braz J Infect Dis 2010;14(5):457-461] @Elsevier Editora Ltda.

\section{INTRODUCTION}

Hepatitis C virus (HCV) infection is currently one of the main causes of hepatocellular carcinoma (HCC) in Western countries. ${ }^{1,2}$ Once HCV-related cirrhosis develops, HCC occurs at an annual rate of $1 \%-4 \%$, although studies from Japan usually report higher rates. ${ }^{3,4}$ In patients with HCV cirrhosis or bridging fibrosis, interferon (IFN) based therapy can achieve an overall rate of sustained virologic response (SVR) ranging from as low as $5 \%$ to almost $50 \%$, depending on the therapeutic regimen used..$^{5-9}$ There is some evidence that IFN treatment can protect against the risk of HCC in HCV cirrhotic patients. ${ }^{10}$ This effect was reported in a randomized controlled trial, where 90 patients with compensated HCV cirrhosis received either $6 \mathrm{MU}$ of IFN-alpha three times a week for 24 weeks or symptomatic treatment. After a mean follow-up of 8.7 years, HCC was found, respectively, in $27 \%$ vs. $73 \%$ of treated and non-treated individuals. Recently, several meta-analysis ${ }^{11-14}$ have confirmed the beneficial effect of IFN in reducing the risk of HCC in HCV cirrhotic patients. However, it remains unclear if this protective effect is related to intrinsic IFN properties or if it is mainly restricted to patients with SVR. Therefore, the aim of the present study was to compare the rate of HCC development in a cohort of HCV cirrhotic patients with versus without SVR after IFN based treatment.

\section{METHODS}

\section{Study design and data collection}

A cohort study was conducted at the Hospital de Clínicas de Porto Alegre, Brazil, a
Authors

Nelson Cheinquer ${ }^{1,2}$

Hugo Cheinquer ${ }^{1,2}$

Fernando H Wolff $2,3,4$

Silvia Coelho-Borges ${ }^{1,2}$

${ }^{1}$ Postgraduate Medical

Education Program

- Gastroenterology,

Faculdade de Medicina,

Universidade Federal do

Rio Grande do Sul, Porto

Alegre, RS, Brazil.

${ }^{2}$ Gastroenterology Service,

Hospital de Clínicas de Porto Alegre, RS, Brazil.

${ }^{3}$ Postgraduate Medical

Education Program

- Epidemiology,

Faculdade de Medicina,

Universidade Federal do

Rio Grande do Sul, Porto Alegre, RS, Brazil.

${ }^{4}$ Instituto para Avaliação de Tecnologia em Saúde (IATS/CNPq), Hospital de Clínicas de Porto Alegre, Universidade Federal do Rio Grande do Sul, Porto Alegre, RS Brazil.

Submitted on: 03/08/2010 Approved on: 05/07/2010

Correspondence to: Fernando H. Wolff, MD, $\mathrm{PhD}$

Rua Dr. Freire Alemão, 351 /703, Porto Alegre, RS, Brazil

CEP: 90450-060 Phone/fax: +55-51-

$30296136 /+55-51$ 92490979

E-mail:

fhwolff@terra.com.br

We declare no conflict of interest. 
tertiary center of the Brazilian public Health System. Patients came to our Viral Hepatitis Outpatient Clinic referred from primary and secondary care centers after the diagnosis of chronic hepatitis C. The routine evaluation of these patients includes HCV-RNA detection, genotyping and quantification, hematological and biochemical tests, and serologic testing for hepatitis B virus and HIV, and an ultrasound guided percutaneous liver biopsy. While on interferon based treatments, patients were followed clinically and with biochemical and hematological tests every four weeks. HCV-RNA testing was performed before and during treatment. All patients were also tested at least one time six or more months after the end of treatment. Hepatic ultrasound scan and serum alpha-fetoprotein testing were performed every six month in cirrhotic patients. Standardized clinical record forms were carefully filled in at patients' routine visits. Baseline and follow-up data were retrospectively collected from those forms.

\section{Participants}

All patients 18 years or older with histologically proven HCV cirrhosis (Metavir 4 or Ishak 5-6) ${ }^{15}$ that have been treated with interferon-based therapies until July 2003 were eligible to the study. Patients who completed treatment after July 2003 were not included in this analysis, in order to have enough follow-up time. All patients were treated with IFN alpha (either conventional or pegylated) plus ribavirin. Genotype 1 patients were treated for 48 weeks, and genotype 2 or 3 patients were treated for 24 weeks according to the main guidelines used at that time.

Patients were excluded from the study if at least one of the following criteria was present: co-infection with hepatitis B or human immunodeficiency virus; diagnosis of other forms of liver disease; alcohol consumption above $10 \mathrm{~g} / \mathrm{d}$; alpha-fetoprotein level greater than $20 \mathrm{ng} /$ $\mathrm{mL}$ at the baseline visit; detection of a focal liver lesion at baseline ultrasound scan or during the first year after treatment. This last criterion was adopted in order to prevent patients who already had HCC to be considered as an incident case. Patients with unstable liver disease were not studied, since these patients were not considered for interferon-based treatments at that time.

\section{Variable definitions and outcomes}

SVR was defined as an undetectable HCV-RNA for more than 24 weeks after the end of therapy. HCV-RNA was measured by a commercial PCR assay, with a lower limit of detection of $50 \mathrm{IU} / \mathrm{mL}$.

The primary endpoint was the development of HCC-based on liver biopsy and/or one of the following: 1) radiologic criteria: focal liver lesion greater than $2 \mathrm{~cm}$ detected in two imaging techniques, (computerized tomography and magnetic resonance imaging) with hepatocellular carcinoma characteristics (arterial hypervascularization, wash-in and wash-out);2) combined criteria: computerized tomography or magnetic resonance imaging with hepatocellular carcinoma characteristics and alpha fetoprotein concentration above $400 \mathrm{ng} / \mathrm{mL}^{16}$

\section{Sample size and statistical analysis}

The study was designed to have a sample size of at least 34 patients with SVR and 34 without SVR. This 68 patients sample would be sufficient to detect a relative risk of 10 with alpha of 0.05 and $80 \%$ power, given an incidence of outcome of 3\% in non-exposed (with SVR) and 30\% in exposed (without SVR). The sample size was increased to include all available patients. StatCalc, Epi-Info 3.3.2 software, was used for sample size calculation.

Data were entered using the Epi-Info 3.3.2 software. The Statistical Package for the Social Sciences (SPSS 13.0, Chicago, Il) was used for statistical analyses. Comparisons of means of continuous variables used t-tests based on linear regression models, adjusted for baseline measurements. P-values are based on two-sided tests and values $<0.05$ were considered significant.

\section{Ethical aspects}

The study was conducted in accordance with the ethical principles of the Declaration of Helsinki, and was approved by the local Ethics Committee. All investigators signed a confidentiality agreement to maintain confidentiality of the information obtained from the review of patients' files.

\section{RESULTS}

Among the $85 \mathrm{HCV}$ cirrhotic patients included in this study, $66 \%$ were male, and mean age was $51.9 \pm 9.6$ years (range 32-72 years). Thirty eight patients (45\%) achieved SVR, while 47 (55\%) were non-responders or relapsers. The mean follow-up time, measured from the last IFN dose up to the last recorded visit was $32.1 \pm 20$ months (range: 12-84 months) among patients with SVR and $28.2 \pm 18$ months (range: 12-96 months) among patients without SVR. Baseline characteristics of the groups with and without SVR are shown in Table 1. All patients had at least one course of treatment with IFN (conventional or pegylated) plus RBV, and 17 (20\%) received a second course of treatment.

Occurrence of HCC was observed in $1(3 \%)$ of 38 patients with SVR, and in $8(17 \%)$ out of the 45 patients without SVR (OR 0.13; 95\% CI 0.006-0.9, $\mathrm{p}=0.02$ ). Mean time interval between the end of therapy and HCC diagnosis was 24 months in the patient with SVR, and 38.3 months in patients without SVR. 
Table 1. Demographical and clinical characteristics of patients with versus without SVR

\begin{tabular}{lccc}
\hline & SVR & Non-SVR & p value \\
& $\mathbf{n = 3 8}$ & $\mathbf{n = 4 7}$ & 0.5 \\
\hline Mean age, years $( \pm$ SD) & $51.1 \pm 8.3$ & $52.6 \pm 10.5$ & 0.4 \\
\hline Male gender $(\%)$ & $24(63)$ & $32(68)$ & 0.04 \\
\hline HCV Genotype 1 (\%) & $5 / 32(16)$ & $16 / 39(41)$ & 0.99 \\
\hline Duration of infection, years $( \pm)$ & $22.2 \pm 6.3^{\ddagger}$ & $22.5 \pm 7.1^{\S}$ & \\
\hline
\end{tabular}

SVR, Sustained Virological Response; SD, Standard Deviation; HCV, Hepatitis C Virus.

Duration of infection defined as interval between exposure to risk factor (whenever identified) and treatment initiation.

${ }^{\ddagger}$ Data available for 24 patients; ${ }^{\circledR}$ Data available for 30 patients.

Figure 1: Cumulative incidence of hepatocellular carcinoma in patients with and without SVR.

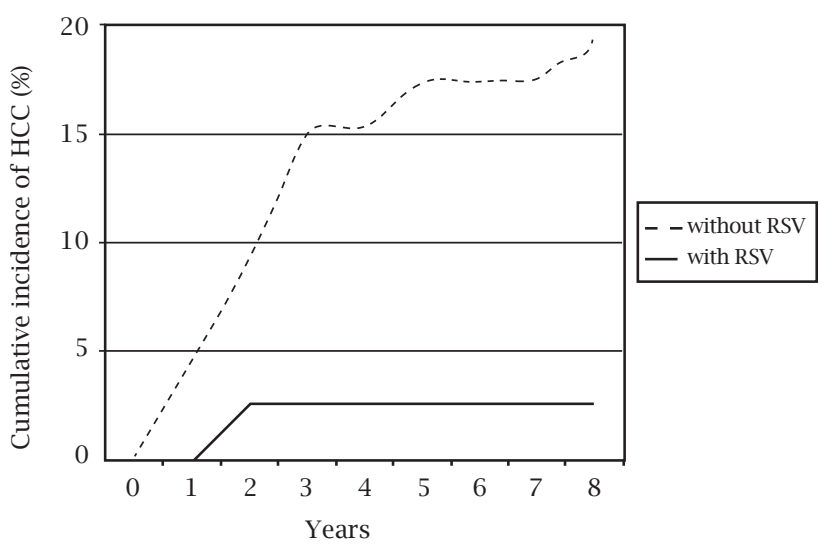

SVR, sustained virological response; HCC, hepatocellular carcinoma.

Figure 1 illustrates the cumulative incidence of hepatocellular carcinoma in patients with and without SVR.

Attributable risk (AR) may be calculated from the HCC risk differences between SVR and non-SVR groups, leading to an etiologic fraction of 14\% (95\% confidence interval: 2.5-26.3). The attributable risk in exposed individuals $(\% \mathrm{AR}=[$ (risk in exposed - risk non-exposed $) /$ risk in exposed] $\mathrm{x} 100)$ is $78 \%$.

\section{DISCUSSION}

Our data, based on a cohort of chronic hepatitis $\mathrm{C}$ cirrhotic patients previously treated with interferon and ribavirin, showed a significant reduction on HCC risk among those who achieved sustained virological response. The attributable risk in exposed individuals, the percentage of the total risk of HCC among patients without SVR that is attributable to not reaching SVR, shows how important SVR is in the prevention of HCC. It is correct to believe, based on our data, that if all patients would have reached SVR, $78 \%$ of the HCC in this population could be prevented. ${ }^{17}$
The rationale behind our study is that the risk of HCC in patients with chronic viral hepatitis correlates with clinical and histological signs of disease severity and increased liver cell proliferation..$^{18}$ Based on this, it has been argued that long-term suppression of viral replication could reduce hepatocyte turnover and liver cell inflammation, leading to a lower risk of dysplasia and cancer. ${ }^{14}$ As IFN alpha combines antiviral, anti-inflammatory, antifibrotic, and antiproliferative properties, it is conceivable that this agent has an intrinsic effect in primary chemoprevention of HCC in patients with chronic viral hepatitis or cirrhosis, regardless of the treatment response.

Several studies were performed to investigate the relationship between IFN-based therapy and HCC development in patients with HCV infection. ${ }^{19-32}$ These studies were usually based on retrospective analysis of small cohorts or small randomized controlled trials (RCT) and showed a marked degree of heterogeneity, making it difficult to assess the actual level of benefit obtained by IFN treatment.

To better evaluate whether IFN reduces the incidence of HCC in patients with HCV cirrhosis, a previous meta-anal$\mathrm{ysis}^{13}$ was updated to include three randomized and 17 nonrandomized controlled trials published up to 2005, totalizing 4,659 patients. $^{14}$ The results showed that IFN decreased HCC rate in all but one of the trials, with a significant difference being observed in 13 of them. Evaluating only studies with consistent results, the authors found that for each 10 patients treated with INF-based regimens, one HCC could be avoided $(\mathrm{NNT}=10)$, including responders and non-responders. The NNT was reduced to 6 when the analysis was restricted to patients who had a sustained response, either biochemical or virological. These findings are in agreement with our results, supporting the hypothesis that the effect of IFN treatment on HCC rate is more related to treatment response than to any direct effect of IFN on carcinogenesis. In the present study a NNT of 7 was found among HCV cirrhotic patients who achieved SVR.

Even though our study was based on a historical cohort, we included patients with similar baseline characteristics. 
Thus, the reduced rate of HCC occurrence observed among treatment responders cannot be ascribed to demographical or clinical differences: all patients were Child-Pugh A, without any past or present history of hepatic imbalance, and showed similar age and gender distribution. Nevertheless, the retrospective design of this study prevents unfolding the contribution of other existing risk factors for HCC, such as aflatoxin exposure, occult HBV infection and/or surreptitious alcohol use..$^{33-36}$

We noted a high incidence of HCC development among the group of patients without SVR (8 out of $45 ; 17 \%$ ). This increased HCC rate could be explained in part by the fact that all patients were submitted to a stringent screening protocol. Nevertheless, this finding is not unusual, and is well inside the range found by other authors which noted HCC occurrence among HCV cirrhotic patients to vary from as low as $7 \%{ }^{23}$ to as high as $38 \%{ }^{19}$ during similar follow-up periods.

In the present study we detected only one case of HCC in the SVR group, during mean follow-up of almost three years. This finding, although encouraging, cannot be overestimated. Indeed, longer periods of follow-up will be required to demonstrate that SVR is truly capable of halting the carcinogenic process in the cirrhotic liver, instead of merely delaying it. In this regard, a recent study from Japan ${ }^{37}$ followed 142 chronic HCV infected patients who achieved SVR. After a mean of approximately five years, de novo HCC was diagnosed in six patients (4.2\%). This data is similar to the $3 \%$ occurrence of HCC described in our study.

In conclusion, we found a beneficial effect of SVR in reducing HCC occurrence in a group of HCV cirrhotic patients treated with the combination of IFN plus RBV, indicating that the presence of the viral agent is probably more important for carcinogenesis than cirrhosis itself. These findings should encourage health care givers to strongly consider HCV eradication, even in the presence of cirrhosis, despite known difficulties inherent to IFN-based treatment in this group of patients with advanced disease. Further studies with a prospective design, longer follow-up, and larger sample size are needed to establish the true role of $\mathrm{HCV}$ eradication in preventing HCC development.

\section{REFERENCES}

1. Llovet JM, Burroughs A, Bruix J. Hepatocellular carcinoma. Lancet 2003; 362: 1907-17.

2. McGlynn KA, El Serag HB. Global epidemiology of hepatocellular carcinoma. In: Bruix J. Hepatocellular Carcinoma. Barcelona: Permanyer, 2005; 1-21.

3. Fattovich G, Stroffolini T, Zagni I, Donato F. Hepatocellular carcinoma in cirrhosis: incidence and risk factors. Gastroenterology 2004; 127:S35-S50.

4. Benvegnu L, Gios M, Boccato S, Alberti A. Natural history of compensated viral cirrhosis: a prospective study on the incidence and hierarchy of major complications. Gut 2004; 53:744-9.
5. Poynard T, Marcellin P, Lee SS et al. Randomized trial of interferon-alfa- $2 b$ plus ribavirin for 48 weeks or for 24 weeks versus interferon-alfa- $2 b$ plus placebo for 48 weeks for treatment of chronic infection with hepatitis C virus. Lancet 1998; 352:1426-30.

6. McHutchison JG, Gordon SC, Schiff E et al. Interferon-alfa-2b alone or in combination with ribavirin as initial treatment for chronic hepatitis C. N. Engl J Med 1998; 339:1485-9.

7. Fried MW, Shiffman ML, Reddy KR et al. Peginterferon-alfa- $2 a$ plus ribavirin for chronic hepatitis $\mathrm{C}$ virus infection. $\mathrm{N}$ Engl J Med 2002; 347:975-82.

8. Manns MP, McHutchison JG, Gordon SC et al. Peginterferonalf $a-2 b$ plus ribavirin compared with interferon-alfa- $2 b$ plus ribavirin for initial treatment of chronic hepatitis $C$ : a randomised trial. Lancet 2001; 358:958-65.

9. Heathcote EJ, Shiffman ML, Cooksley WG et al. Peginterferonalfa-2a in patients with chronic hepatitis $\mathrm{C}$ and cirrhosis. $\mathrm{N}$ Engl J Med 2000; 343:1673-80.

10. Nishiguchi S, Shiomi S, Nakatani S et al. Prevention of hepatocellular carcinoma in patients with chronic active hepatitis $\mathrm{C}$ and cirrhosis. Lancet 2001; 357:196-7.

11. Poynard T, Moussalli J, Ratziu V, et al. Is antiviral treatment (IFN-alpha and/or ribavirin) justified in cirrhosis related to hepatitis C virus? Acta Gastroenterol Belg 1998; 61:431-37.

12. Papatheodoridis GV, Papadimitropoulos V, Hadzyiannis SJ. Effect of interferon therapy on the development of hepatocellular carcinoma in patients with hepatitis $\mathrm{C}$ virus-related cirrhosis: a meta-analysis. Aliment Pharmacol Ther 2001; 15:689-98.

13. Cammà $\mathrm{C}$, Giunta $\mathrm{M}$, Andreone $\mathrm{P}$, Craxi $\mathrm{A}$. Interferon and prevention of hepatocellular carcinoma in viral cirrhosis: an evidence-based approach. J Hepatol 2001; 34:593-602.

14. Craxi A, Cammà C. Prevention of hepatocellular carcinoma. Clin Liv Dis 2005; 9:329-46.

15. Bedossa P, Poynard T. An algorithm for the grading of activity in chronic hepatitis C. The METAVIR Cooperative Study Group. Hepatology 1996; 24:289-93.

16. Bruix J, Sherman M, Llovet, et al. Clinical management of hepatocellular carcinoma. Conclusions of the Barcelona-2000 EASL conference. J Hepatol 2001; 35:421-30.

17. Szklo M, Nieto FJ. Measuring Associations between Exposures and Outcomes. In: Szklo M, Nieto FJ, eds. Epidemiology: Beyond the Basics. $2^{\text {nd }}$ ed. Jones and Bartlett Publishers, 2007: 77-104.

18. Thorgeirsson SS, Grisham JW. Molecular pathogenesis of hepatocellular carcinoma. Nat Genet 2002; 31:339-46.

19. Nishiguchi S, Kuroki T, Nakatani S, et al. Randomised trial of effects of interferon-alfa on incidence of hepatocellular carcinoma in chronic active hepatitis C with cirrhosis. Lancet 1995; 346:1051-5.

20. Mazzella G, Accogli E, Sottili S, et al. Alpha-interferon treatment may prevent hepatocellular carcinoma in HCV-related liver cirrhosis. J Hepatol 1996; 24:141-7.

21. International Interferon-alpha Hepatocellular Carcinoma Study Group. Effect of interferon-alpha on progression of cirrhosis to hepatocellular carcinoma: a retrospective cohort study. Lancet 1998; 351:1535-9.

22. Imai Y, Kawata S, Tamura S, et al. for the Osaka Hepatocellular Carcinoma Prevention Study Group. Relation of interferon therapy and hepatocellular carcinoma in patients with chronic hepatitis C. Ann Intern Med 1998; 129:94-9

23. SerfatyL, Aumaître H, Chazouillères $\mathrm{O}$, et al. Determinants of outcome of compensated hepatitis $\mathrm{C}$ virus-related cirrhosis. Hepatology 1998; 27:1435-40. 
24. Sofia S, Casali A, Buscarini E. Effect of lymphoblastoid IFN in the treatment of liver cirrhosis and prevention of HCC. Ital. J. Gastroenterol Hepatol 1998; 30:A31.

25. Benvegnù L, Chemello L, Noventa F, et al. Retrospective analysis of the effect of interferon therapy on the clinical outcome of patients with viral cirrhosis. Cancer 1998; 83:901-9.

26. Shioda A, Moriyama M, Kaneko M. Long-term prognosis of hepatocellular carcinoma developing after treatment of interferon in patients with chronic hepatitis $\mathrm{C}$ and liver cirrhosis. Hepatology 1999; 30:268A.

27. Yoshida H, Shiratori Y, Moriyama M, et al. Interferon therapy reduces the risk for hepatocellular carcinoma: national surveillance program of cirrhotic and noncirrhotic patients with chronic hepatitis C in Japan. Ann Intern Med 1999; 131:174-81.

28. Mura D, Deliperi R, Fastame L. Five year follow-up after Interferon therapy in HCV-positive compensated cirrhosis. Ital J.Gastroenterol Hepatol 1998; 30:A114.

29. Valla D-C, Chevallier M, Marcellin P, et al. Treatment of hepatitis $\mathrm{C}$ virus-related cirrhosis: a randomized controlled trial of interferon-alfa-2b versus no treatment. Hepatology 1999; 29:1870-5.

30. Ikeda K, Saitoh S, Suzuki Y, et al. Interferon decreases hepatocellular carcinogenesis in patients with cirrhosis caused by the hepatitis B virus. Cancer 1998; 82:827-35.
31. Kasahara A, Hayashi N, Mochizuki K, et al. Risk factors for hepatocellular carcinoma and its incidence after interferon treatment in patients with chronic hepatitis C. Hepatology 1998; 27:1394-402.

32. Miyajima I, Sata M, Kumashiro R, et al. The incidence of hepatocellular carcinoma in patients with chronic hepatitis $\mathrm{C}$ after interferon treatment. Oncol Rep 1998; 5:201-4.

33. Ikeda K, Saitoh S, Koida I, et al. A multivariate analysis of risk factors for hepatocellular carcinogenesis: a prospective observation of 795 patients with viral and alcoholic cirrhosis. Hepatology 1993; 18:47-53.

34. International Interferon-alpha Hepatocellular Carcinoma Study Group. Effect of interferon-alpha on progression of cirrhosis to hepatocellular carcinoma: a retrospective cohort study. Lancet 1998; 351:1535-9.

35. Ghebranious N, Sell S. Hepatitis B injury, male gender, aflatoxin, and p53 expression each contribute to hepatocarcinogenesis in transgenic mice. Hepatology 1998; 27:383-439.

36. Sun Z, Lu P, Gail MH, et al. Increased risk of hepatocellular carcinoma in male hepatitis B surface antigen carriers with chronic hepatitis who have detectable urinary aflatoxin metabolite M1. Hepatology 1999; 30:379-83.

37. Yamaura T, Matsumoto A, Rokuhara A, et al. Development of small hepatocellular carcinoma in a patient with chronic hepatitis $\mathrm{C}$ after 77 months of a sustained and complete response to interferon therapy. J Gastroenterol Hepatol 2002; 17:1229-35. 